\section{Valoración de la actividad física en adolescentes escolares por medio de cuestionario}

\author{
Assessment of physical activity \\ in adolescent students \\ through questionnaire
}

\begin{abstract}
Objectives: To compare parameters of physical activity (PA) among school age adolescents of both sexes and propose evaluation curves to estimate physical activity levels in students of an educational establishment of the Municipality of Talca, Chile. Subjects and methods: The type of study was descriptivecomparative. 1448 adolescent students (749 women and 699 men) from the municipal School of Excellence "Abate Molina" in Talca were studied. Age range was 12.0 to 18.9 years. Physical activity was assessed by a questionnaire including 11 questions. The LMS method was used to estimate centiles valuation ranging from p3, p10, p25, p50, p75, p90 and p97. Results: The questionnaire showed a Cronbach's alpha of $r=0.82$ in men and $r=0.79$ in women. Males achieved a score of $28.46 \pm 6.88$ and women $23.88 \pm 5.69$. The breakpoints adopted for levels of physical activity are: $<p 3$ very low level of $P A ;$ p3-p25 low level $P A ; \quad p 25-p 75$ moderate level of PA; p75-p97 high level of PA and $>97$ very high level of $P A$. Conclusion: Female adolescents showed lower levels of PA in relation to males. The curves for assessing PA are an alternative to be applied in the School "Abate Molina" as part of routine physical education classes and its use could be extended to other schools in the Municipality of Talca. Key words: Physical activity, teenagers, weight curves, physical activity questionnaire.
\end{abstract}

Aquiles Yáñez-Silva (1)

Jefferson Eduardo Hespanhol (2)

Rossana Gómez Campos $(3,4,5)$

Marco Cossio-Bolaños $(6,7)$

(1) Facultad de Ciencias Básicas. Universidad Católica del Maule, Talca, Chile. (2) Faculdade de Educação Física. Pontifícia Universidade Católica de Campinas, SP, Brasil. (3) Universidad Autónoma de Chile, Chile. (4) Grupo de Estudios Interdisciplinar en Ciencias de la Salud y Deporte, GEISADE, Universidad Autónoma de Chile, Talca, Chile. (5) Faculdade de Educação Física. Universidade Estadual de Campinas, SP, Brasil. (6) Departamento de Ciencias de la Actividad Física. Universidad Católica del Maule, Talca, Chile. (7) Centro de Investigación en Desarrollo Biológico Humano CIDEBIHU, Universidad Católica del Maule, Talca, Chile.

Dirigir la correspondencia a: Profesor

Marco Cossio-Bolaños Departamento de Ciencias de la Actividad Física Universidad Católica del Maule Talca, Chile

E-mail: mcossio1972@hotmail.coM

Este trabajo fue recibido el 14 de Junio de 2014 y aceptado para ser publicado el 8 de Septiembre de 2014.

\section{INTRODUCCIÓN}

La actividad física (AF) es una conducta o más apropiadamente una serie de conductas que implican movimientos corporales producidos por los músculos esqueléticos (1). Constituye en gran parte a la promoción de la salud y básicamente en la prevención de enfermedades crónicas no-transmisibles. Su valoración generalmente es abordada desde varios puntos de vista, a partir de métodos cuantitativos y/o cualitativos o también denominados métodos directos (agua doblemente marcada, calorimetría directa, sensores de movimiento, acelerómetros, diarios de AF y observación directa) y/o indirectos (VO2max, frecuencia cardiaca, cuestionarios e informes de cantidad de ingestión de energía).

De cualquier forma, independientemente del método a utilizar, un bajo índice de AF es considerado como un predictor de enfermedades cardiovasculares, no sólo en escolares con sobrepeso u obesidad, sino también en escolares eutróficos (2).
De hecho, los cuestionarios denominados métodos indirectos y/o cualitativos son la forma más utilizada para valorar la $A F$, en razón a su bajo costo y por la facilidad en su uso y en la administración del instrumento a grandes grupos poblacionales en un sólo momento. La Organización Mundial de la Salud (3) realizó algunas recomendaciones mundiales en cuanto a los instrumentos de medición, en el que deben abordar los indicadores de frecuencia, duración, intensidad, tipo y cantidad total de AF. En varios de sus informes reconocen que la AF en general es un factor determinante en el consumo de energía, por lo que es fundamental para conseguir el equilibrio energético y el control del peso $(4,5)$.

El papel de la AF durante la etapa del crecimiento y del desarrollo es relevante, debido a sus numerosos beneficios relacionados con la salud física y psicológica (6). La adolescencia es una etapa de la vida que se caracteriza por ser un periodo en el cual se producen intensos cambios biológicos, 
psicológicos y cognitivos (7).

La valoración de AF puede ser utilizada para desarrollar investigaciones transversales, longitudinales y/o experimentales, así como para proporcionar información a las autoridades gubernamentales para que puedan generar políticas públicas a nivel local, regional y nacional. En los últimos años a nivel internacional se han realizado varios estudios relacionados con la AF en niños y adolescentes (8-10). Tales investigaciones, independientemente del tipo de método utilizado, fueron diseñadas para determinar los niveles de AF y verificar las diferencias por sexo, así como para estimar la proporción de la población que logra un nivel de umbral activo y/o inactivo en relación a la salud, sin embargo, a nivel nacional son escasos los estudios que valoran los patrones de AF durante la infancia y nulos durante la adolescencia, inclusive se desconoce sobre la disparidad de los niveles de AF entre adolescentes. Chile no cuenta a nivel nacional y regional con normativas y/o criterios de referencia para la valoración de los patrones de AF en escolares, por lo que es una necesidad urgente proponer criterios de valoración que permitan diagnosticar, monitorizar y vigilar los patrones de AF en adolescentes escolares de ambos sexos de la comuna de Talca, lo que podría facilitar la labor de los profesores de educación física en los centros escolares y ser aplicado rutinariamente con el único propósito de identificar a los escolares más o menos activos.

El objetivo de este estudio fue comparar los parámetros de actividad física de adolescentes escolares por sexo y proponer curvas de valoración para estimar los niveles de AF de un establecimiento educativo de la red municipal de Talca.

\section{SUJETOS Y MÉTODOS}

Tipo de estudio y muestra

El presente estudio fue de tipo descriptivo comparativo. Se estudiaron 1448 adolescentes escolares (749 mujeres y 699 hombres) del Colegio Municipalizado de Excelencia "Abate Molina" de la comuna de Talca (Chile). El rango de edad osciló entre 12,0 y 18,0 años a la fecha de la evaluación. Los escolares realizaban actividad física una vez por semana (90 min/sesión), como parte de las clases de educación física. La selección de la muestra fue de tipo no-probabilística (por cuotas). La tabla 1 ilustra el tamaño de la muestra de escolares estudiada. Del total de alumnos de dicha institución educativa, se evaluó al 69\% de los escolares.En general, la economía se basa principalmente en la agricultura y la ganadería, siendo relevante la actividad vitivinícola.

Se incluyeron en el estudio a los escolares cuyos padres y/o apoderados autorizaron la ficha de consentimiento y a los que se encontraban clínicamente sanos al momento de la evaluación antropométrica. Se excluyó al 28\% de escolares que no autorizaron el consentimiento y al 3\% de los escolares que se ausentaron durante los días de recolección de datos (total 31\%). Todos los padres y apoderados fueron informados sobre el proceso de evaluación de las medidas antropométricas y la aplicación de un cuestionario para recopilar información respecto a la actividad física. El estudio contó con la respectiva autorización del Director de la Institución Educativa y del Comité de Etica local. Todo el proceso de recolección estuvo de acuerdo a las normas establecidos por la Declaración de Helsinki.

\section{Procedimientos}

El proceso de recolección de datos se efectuó durante los meses de setiembre y octubre de 2013. Las evaluaciones se efectuaron en horario de clases antes de realizar cualquier esfuerzo físico. Inicialmente se aplicó el cuestionario y posteriormente las medidas antropométricas. La recolección de datos estuvo a cargo de 4 profesionales altamente entrenados.

La edad cronológica se determinó a nivel decimal, para ello se utilizó la fecha decimal del día, mes y año de nacimiento, así como la fecha de la evaluación.

La valoración de la AF se efectuó mediante la técnica de la encuesta, aplicándose un cuestionario con 11 ítems de medición propuesto por Gómez-Campos et al (11). Los adolescentes dispusieron entre 10 a 15 minutos para responder a las cuestiones señaladas mediante alternativas múltiples. El cuestionario fue respondido en sus respectivas clases según curso y horario. La puntuación mínima es de 11 puntos y la puntuación máxima es de 45 puntos. El instrumento en este estudio mostró una capacidad de reproductibilidad de alfa de Cronbach de ( $r=0,82$ en hombres y $r=0,79$ en mujeres). Para calcular el Error Técnico de Medida (ETM) intra-evaluador e inter-evaluador se evaluó el cuestionario en dos oportunidades (test re-test) con un intervalo de 6 días. Los valores oscilaron entre $3-4 \%$, respectivamente.

\section{TABLA 1}

Tamaño de la muestra de escolares por curso y sexo.

\section{Cursos}

Mujeres

n

50

48

$8^{\circ}$ año

Educación media

$1^{\circ}$ año

$2^{\circ}$ año

$3^{\circ}$ año

4 año

Total
120

188

194

149

749
6,7

6,4

16,0

25,1

25,9

19,9

100,0
Varones

n $\%$

67

9,6

9,6

67

13,3

24,3

23,5

19,7

100,0 
Para la evaluación de las variables antropométricas se adoptó el protocolo estandarizados por el "international working group of kineanthropometry" descrita por Ross y Marfell-Jones (12). Se evaluó el peso corporal (kg) descalzo y con la menor cantidad de ropa posible utilizando una balanza digital con una precisión de (100g) de marca Tanita y con una escala de (0 a $150 \mathrm{~kg}$ ). La estatura $(\mathrm{cm})$ se evaluó de acuerdo al plano de Frankfurt sin zapatos utilizando un estadiómetro de aluminio de marca Seca graduada en milímetros con una escala de $(0-250 \mathrm{~cm})$. Se calculó el Índice de Masa Corporal IMC (kg/ $\mathrm{m} 2$ ) con la fórmula de Quetelet (13), donde [IMC=Peso(kg)/ Estatura(m)2]. El 10\% de la muestra total fue seleccionada aleatoriamente para ser evaluado en dos oportunidades, tanto en peso, como en estatura. A través de estas mediciones se calculó el Error Técnico de Medida (ETM) intra-evaluador e inter-evaluadores. Los porcentajes oscilaron entre 1 a $3 \%$, respectivamente.

\section{Análisis estadístico}

La distribución normal fue verificada a través de la prueba Kolmogorov Smirnov. Esto se aplicó a cada variable y por separado. Los resultados fueron analizados por medio de la estadística descriptiva de media, desviación estándar y distribución percentilar (p3, p10, p25, p50, p75, p90 y p97). Los valores medios de los hombres y mujeres fueron comparados a través de la prueba de " $\mathrm{t}$ " student para muestras independientes $(p<0,001)$.

Curvas de percentiles para la valoración de la AF por edad y sexo fueron construidos a través del método LMS (14). Para normalizar los datos para cada edad se tuvo que realizar una transformación de potencia de Box-Cox. Se aplicó el procedimiento de máxima probabilidad de penalización a efecto de crear tres curvas suaves: $L(t)$ Box-Cox power, $M(t)$ mediana $y$ $\mathrm{S}(\mathrm{t})$ Coeficiente de variación. Estos tres parámetros permiten construir la curva en relación a cualquier centil que se desee, para ello es necesario el uso de la fórmula: $C 100 \alpha(t)=M(t)$ $[1+L(t) S(t) Z \alpha] 1 / L(t)$, donde Z $\alpha$ es el desvío normal equivalente para el área $\alpha, C 100 \alpha(t)$ es el centil correspondiente al $\mathrm{Za}$ equivalente a los grados de libertad para $L(t), M(t)$ y $S(t)$ que miden la complejidad de cada curva ajustada. El método LMS se aplicó para generar patrones de AF con el software LMS Chartmaker Pro versión 2.3 (15).

\section{RESULTADOS}

Las variables antropométricas que caracterizan a la muestra estudiada se observan en la tabla 2. No hubo diferencias en la edad y en el IMC cuando fueron comparados en función del sexo. Los hombres eran más altos y presentaban mayor peso corporal que las mujeres $(p<0,001)$.

Las comparaciones de los parámetros de AF por edad y sexo se observan en la figura 1. En todas las franjas de edades los hombres presentan valores superiores de AF 28,46 $\pm 6,88$ que las mujeres $(23,88 \pm 5,69)$. No hubo diferencias significativas entre edades en hombres y mujeres, a pesar de que con el transcurso de la edad en ambos sexos, los niveles de AF van disminuyendo lentamente.

La propuesta de percentiles por el método LMS se observa en la tabla 3. En la figura 2 se ilustran las curvas por edad y sexo y distribuidos en percentiles. En ambos casos se interpreta como: <p3 muy bajo nivel de AF, p3-p25 bajo nivel de AF, p25-p75 moderado nivel de AF, p75-p97 alto nivel de AF y >p97 muy alto nivel de AF.

\section{DISCUSIÓN}

Los resultados del estudio evidenciaron que los varones mostraron mayores niveles de AF que las mujeres de los 12 hasta los 18 años. Estos hallazgos son consistentes con otros estudios internacionales (16-18), independientemente del método utilizado y la zona geográfica donde se efectuaron a cabo tales estudios.

Las diferencias por sexo básicamente se atribuyen al contexto social y cultural; tales aspectos, son considerados importantes agentes influyentes sobre las experiencias en la AF en mujeres adolescentes (19). La literatura destaca que existen condicionantes sociales que generalmente se presentan durante la fase crítica de la adolescencia (20) los cuales podrían ser interpretadas como posibles barreras que impiden la realización de AF específicamente en mujeres adolescentes. Algunos estudios internacionales destacan la imagen de sí mismo, la influencia de los amigos y la familia, el tipo de programas de AF para cada sexo, la facilidad en el acceso a las instalaciones, seguridad en las instalaciones (21-24), entre otros aspectos.

En general, independientemente de la edad, sexo y factores socioculturales, los adolescentes en la actualidad están adoptando estilos de vida cada vez más inactivos, lo que favorece al aumento de posibles enfermedades crónicas no-transmisibles a edades cada vez más tempranas. A nivel nacional no fue posible identificar estudios que comparan los niveles de AF por género, sin embargo, consideramos que esta tendencia sea similar en otras regiones del país, puesto que la adolescencia es un foco para la presencia de múltiples riesgos, ya que la mayoría de los comportamientos se desarrollan y se adoptan durante la infancia y a principios de la

\section{TABLA 2}

Peso corporal, estatura e Índice de Masa Corporal (IMC) de los escolares, según sexo.

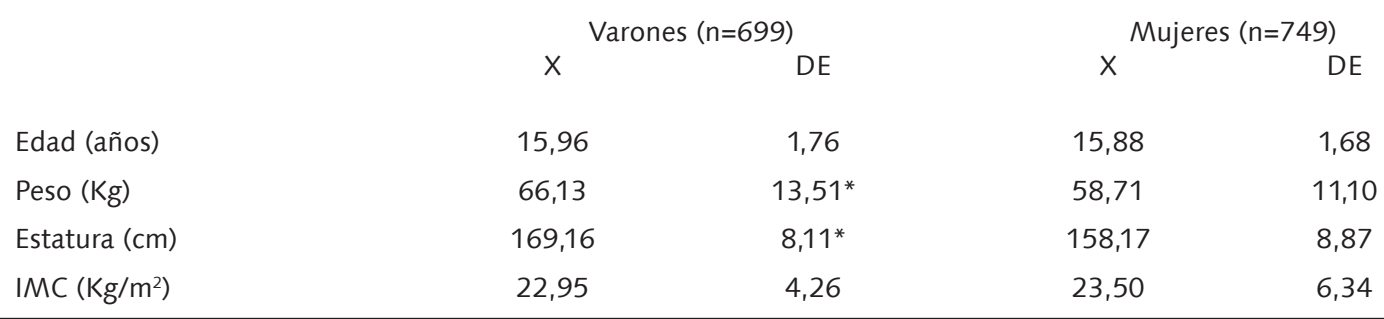

* = significativo en relación a las mujeres $(p<0,001)$. 


\section{FIGURA 1}

Parámetros de AF en función de la edad y sexo.

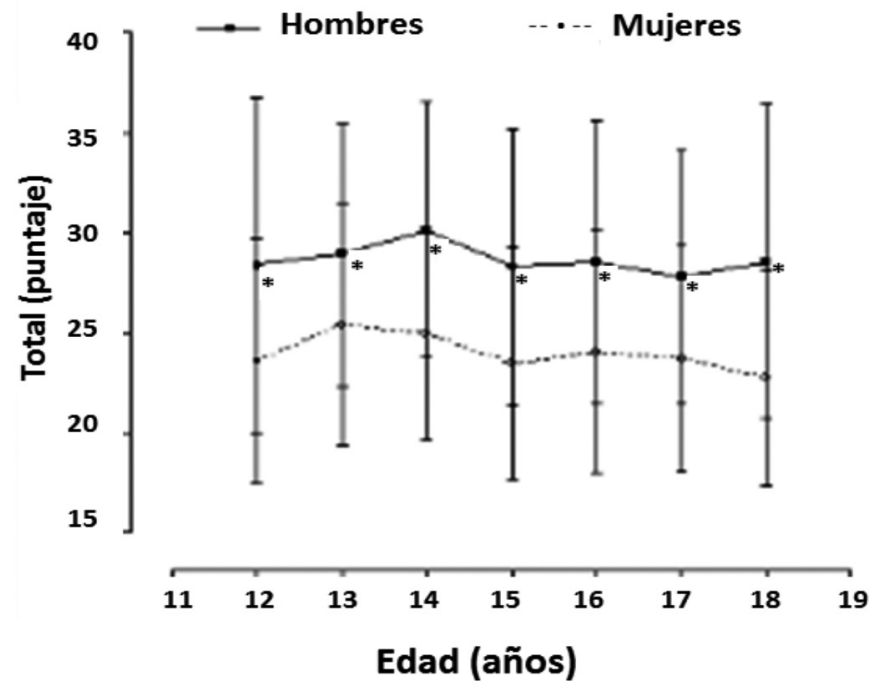

* diferencia significativa en relación a las mujeres ( $<<0,001)$, Puntaje (se refiere al puntaje obtenido a partir de la sumatoria de las 11 preguntas).

\section{TABLA 3}

Valores de L, M y S, y percentiles de AF por edad y sexo.

Edad (años)

\begin{tabular}{|c|c|c|c|c|c|c|c|c|c|c|c|}
\hline & $\mathrm{L}$ & $M$ & $\mathrm{~S}$ & $\mathrm{~N}$ & P3 & P10 & P25 & P50 & P75 & P90 & P97 \\
\hline \multicolumn{12}{|c|}{ Varones $(n=699)$} \\
\hline $12,0-12,9$ & 1,9 & 29,1 & 0,2 & 43 & 13,6 & 19,9 & 24,8 & 29,1 & 32,8 & 36,2 & 39,3 \\
\hline $13,0-13,9$ & 1,7 & 29,7 & 0,2 & 58 & 14,3 & 20,3 & 25,3 & 29,7 & 33,7 & 37,4 & 40,9 \\
\hline $14,0-14,9$ & 1,5 & 29,8 & 0,2 & 56 & 14,7 & 20,3 & 25,3 & 29,8 & 34,1 & 38,0 & 41,8 \\
\hline $15,0-15,9$ & 1,3 & 29,0 & 0,2 & 139 & 14,4 & 19,7 & 24,5 & 29,0 & 33,3 & 37,5 & 41,5 \\
\hline $16,0-16,9$ & 1,2 & 28,5 & 0,2 & 144 & 14,1 & 19,5 & 23,9 & 28,5 & 32,9 & 37,2 & 41,3 \\
\hline $17,0-17,9$ & 1,1 & 28,0 & 0,2 & 157 & 13,9 & 18,3 & 23,4 & 28,0 & 32,6 & 37,0 & 41,5 \\
\hline $18,0-18,9$ & 1,0 & 28,0 & 0,3 & 102 & 13,6 & 18,4 & 23,2 & 28,0 & 32,8 & 37,6 & 42,4 \\
\hline \multicolumn{12}{|c|}{ Mujeres $(n=749)$} \\
\hline $12,0-12,9$ & 0,2 & 24,3 & 0,2 & 42 & 14,9 & 17,6 & 20,8 & 24,3 & 28,3 & 32,7 & 37,7 \\
\hline $13,0-13,9$ & 0,3 & 24,6 & 0,2 & 45 & 14,9 & 17,7 & 21,0 & 24,6 & 28,6 & 33,2 & 38,2 \\
\hline $14,0-14,9$ & 0,3 & 24,2 & 0,2 & 59 & 14,5 & 17,4 & 20,6 & 24,2 & 28,3 & 32,8 & 37,8 \\
\hline $15,0-15,9$ & 0,3 & 23,5 & 0,2 & 168 & 13,9 & 16,7 & 19,9 & 23,5 & 27,5 & 31,9 & 36,8 \\
\hline $16,0-16,9$ & 0,3 & 23,3 & 0,2 & 133 & 13,7 & 16,6 & 19,7 & 23,3 & 27,3 & 31,7 & 36,6 \\
\hline $17,0-17,9$ & 0,4 & 23,1 & 0,2 & 189 & 13,7 & 16,5 & 19,6 & 23,1 & 27,0 & 31,3 & 36,0 \\
\hline $18,0-18,9$ & 0,4 & 22,7 & 0,2 & 113 & 13,7 & 16,4 & 19,4 & 22,7 & 26,4 & 30,4 & 34,8 \\
\hline
\end{tabular}

Nivel de Actividad Física

$\mathrm{L}=$ asimetría; $M=$ mediana; $\mathrm{S}=$ coeficiente de variación; Nivel de Actividad Física (NAF) (obtenido a partir del puntaje total del cuestionario). 
adolescencia $(25,26)$.

A pesar de que varios estudios han evidenciado algunas barreras que impiden la práctica regular de la AF en las adolescentes, se destaca que la disparidad por sexo sigue siendo un tema controversial, puesto que algunos estudios recientes verificaron que los niños a pesar de ser más activos que las niñas, también mostraron comportamientos más sedentarios $(27,28)$. Por lo tanto, es necesario continuar estudiando los patrones de la AF en escolares adolecentes, no sólo en función de la edad y sexo, sino, también a partir de variables sociales, deportivas, culturales, biológicas y psicológicas, los cuales podrían posibilitar una mejor comprensión de los patrones de AF dentro y fuera de la escuela.

Respecto a la valoración de la AF de los adolescentes del estudio, se utilizó el cuestionario propuesto por Gómez-Campos et al (11), el cual valora los niveles de AF en adolescentes escolares. Dicho instrumento aborda vínculos relacionados con la frecuencia, duración, intensidad, tipo y cantidad total de $A F$, por lo que está en estrecha relación con las características descritas por la OMS (3), ya que sugiere considerar directrices respecto a la relación dosis-respuesta entre AF y los beneficios para la salud, sobre todo, para prevenir enfermedades no-transmisibles a partir de las cuatro categorías antes mencionadas.

El instrumento utilizado en este estudio mostró una alta capacidad de reproductibilidad, reflejando un coeficiente de alfa de Cronbach de $(r=0,82)$ en varones y de $(r=0,79)$ en mujeres. Estos valores son similares a otros estudios internacionales, en los que se utilizaron cuestionarios para medir niveles de AF $(29,30)$ y perfil de la salud y enfermedad (31). Además en términos de calidad de medición la confiabilidad es un requisito necesario que debe ser efectuado con la intención de alcanzar una mayor precisión y reproducción de los instrumentos (32), de esta forma, es posible garantizar resultados confiables.
Desde esa perspectiva, ante la necesidad de contar con datos normativos en el Colegio "Abate Molina" de Talca que permitan valorar la AF en los escolares de ambos sexos, los resultados brutos del cuestionario aplicado fueron transformados a valores ordinales, luego se aplicó el método LMS (14) para estimar centiles de valoración que van desde p3, p10, 925, p50, p75, p90 a p97, además se utilizó las transformación de Box-Cox power para normalizar los datos en cada edad y sexo. Los tres parámetros LMS permitieron construir las curvas en relación a cualquier centil a través del software LMS Chartmaker Pro versión 2.3 (15).

Las curvas propuestas son una posibilidad para valorar los niveles de AF en adolescentes, puesto que actualmente la práctica de $\mathrm{AF}$ en niños y adolescentes es cada vez menos frecuente, lo que conlleva a que el sedentarismo se haya convertido en un problema de salud pública (33). Evidentemente, la propuesta de tales curvas son esenciales para la interpretación individual y colectiva de escolares adolescentes, cuyos resultados pueden contribuir eficientemente al diagnóstico, monitoreo y control de los patrones de AF, inclusive, dar la posibilidad de clasificar e identificar a los adolescentes más activos o menos activos, con lo cual, es factible desarrollar estrategias de promoción de la salud en la escuela, y abre la posibilidad de investigar a los escolares que están en riesgo de desarrollar alguna enfermedad crónica y así poder implementar iniciativas dentro del establecimiento o derivarlos a un centro de salud (34).

A pesar de que los cuestionarios son instrumentos subjetivos, cuyas mediciones podrían presentar sesgos tanto, en los encuestados, como en los encuestadores, el presente estudio muestra algunas ventajas relevantes, por ejemplo, presenta valores aceptables de confiabilidad $(r=0,79-0,82)$ y Error Técnico de Medida intra e inter-evaluador (<4\%), así como el método utilizado (LMS) para construir las curvas garantizan adecuados puntos de corte, cuyos percentiles extremos pueden

\section{FIGURA 2}

Curvas de AF por edad y sexo.
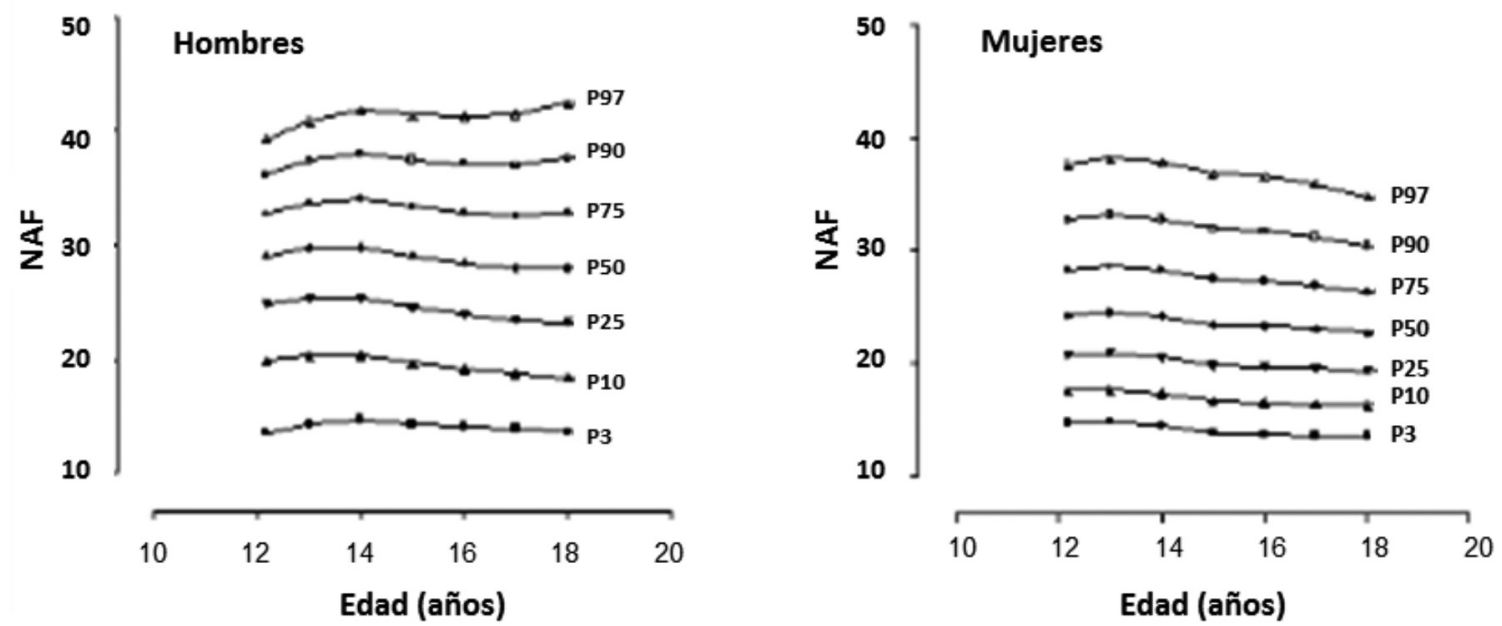

Nivel de Actividad Física NAF. 
ser estimados de manera más eficiente. Se considera relevante la realización de más estudios, en el que se utilicen métodos cuantitativos para poder generar normas de referencia a nivel regional y nacional, así como ampliar el rango de edad, abarcando a niños, adolescentes, jóvenes y adultos. También para estudios futuros se considera oportuno controlar los hábitos de alimentación, el estado nutricional y el gasto energético, puesto que son variables que están en estrecha relación con los niveles de AF y podrían ser pertinentes para controlar de mejor forma el estilo de vida de los adolescentes.

\section{CONCLUSIÓN}

Las adolescentes de sexo femenino evidenciaron menor nivel de AF en relación a los hombres. Las curvas para valorar la AF son una alternativa para ser aplicado en el Colegio "Abate Molina" con posibilidad de ser utilizados en otros colegios, cuyas características sean similares a los del presente estudio. Los resultados sugieren promover mayor AF en el grupo de las adolescentes y los estándares propuestos a partir del cuestionario de AF podrían ser aplicados en la escuela de forma rutinaria.

\section{RESUMEN}

Objetivos: Comparar los parámetros de actividad física (AF) de adolescentes escolares de ambos sexos y proponer curvas de valoración para estimar los niveles de actividad física de un establecimiento educativo de la red municipal de Talca. Sujetos y métodos: El tipo de estudio fue descriptivocomparativo. Se estudiaron 1448 adolescentes escolares (749 mujeres y 699 hombres) del Colegio Municipalizado de Excelencia "Abate Molina" de la comuna de Talca (Chile). El rango de edad oscila entre 12,0-18,9 años. La actividad física (AF) se valoró por medio de un cuestionario que incluía 11 preguntas. Se aplicó el método LMS para estimar los centiles de valoración que van desde p3, p10, p25, p50, p75, p90 y p97. Resultados: El cuestionario mostró un alfa de Cronbach de $r=0,82$ en varones y de $r=0,79$ en mujeres. Los varones alcanzaron un puntaje de $28,46 \pm 6,88$ y las mujeres de $23,88 \pm 5,69$. Los puntos de corte adoptados para los niveles de actividad física fueron: <p3 muy bajo nivel de AF, p3-p25 bajo nivel de AF, p25-p75 moderado nivel de AF, p75-p97 alto nivel de AF y >p97 muy alto nivel de AF. Conclusión: Las adolescentes de sexo femenino evidenciaron menor nivel de AF que los hombres. Las curvas para valorar la AF son una alternativa para ser aplicado en el Colegio "Abate Molina" como parte de la rutina en las clases de educación física y su uso podría extenderse a otros centros escolares de la Comuna de Talca.

Palabras clave: Actividad física, adolescentes, curvas de peso, cuestionario para actividad física.

Agradecimientos: Se agradece a los Profesores de Educación Física: Rosita Aravena Y., Alejandra Álvarez P., Carla Donoso, Williams Cabello, Andrés Flores, Luis Guerrero, Enrique Zúñiga y Margarita Cornejo Silva (Jefe del departamento). A los Inspectores: Eduardo Basualto Adasme y Hugo Arellano Norambuena, por su valiosa colaboración en la organización de los estudiantes. Al Director del Colegio LAM: César López Moreno y Víctor Hugo Muñoz Caroca, jefe de ATP. Al Decano de la Facultad de Ciencias Básicas UCM: Profesor Raúl Becerra Huencho, por su colaboración.

\section{BIBLIOGRAFÍA}

1. Malina RM, Little BB. Physical Activity: The Present in the Context of the Past. Am J. Hum Biol 2008;20:373-91.
2. Wei M, Kampert JB, Barlow CE, Nichaman MZ, Gibbons LW, Paffenbarger RS, et al. Relationship between low cardiorespiratory fitness and mortality in normal-weight, owerweight, and obese men. JAMA. 1999;282:1547-53.

3. World Health Organization: Global Strategy on Diet, Physical Activity and Health. 22 WHA57.17. Geneva, Switzerland: World Health Organization. May 2004.

4. Informe sobre la salud en el mundo 2002: Reducir los riesgos y promover una vida sana. Ginebra, Organización Mundial de la Salud, 2002.

5. The global burden of disease: 2004 update. Geneva, Organización Mundial de la Salud, Ginebra, 2008.

6. Hills AP, King NA, Armstrong TP. The Contribution of Physical Activity and Sedentary Behaviours to the Growth and Development of Children and Adolescents: Implications for Overweight and Obesity. Sports Med 2007; 37 (6): 533-45

7. Stang J, Story M. Adolescent Growth and development. Stang J, Story M (eds) Guidelines for Adolescent Nutrition Services 2005.

8. Fogelholm M, Stigman S, Huisman T, Metsamuuronen J: Physical fitness in adolescents with normal weight and overweight. Scand J Med Sci Sports 2008; 18(2):162-70.

9. Ortega FB: Cardiovascular fitness modifies the associations between physical activity and abdominal adiposity in children and adolescents: the European Youth Heart Study. BJSM 2010, 44:256-62.

10. Rauner $A$, Mess $F$, Woll $A$. The relationship between physical activity, physical fitness and overweight in adolescents: a systematic review of studies published in or after 2000. Pediatrics 2013, 13-9.

11. Gómez-Campos R, Vilcazán E, Arruda M, Hespanhol JE, Cossio-Bolaños MA. Validación de un cuestionario para la valoración de la actividad física en escolares adolescentes. An Fac Med. 2012;73(4):307-13.

12. Ross WD, Marfell-Jones MJ. Kinanthropometry. In: MacDougall JD, Wenger HA, Geen HJ, editors. Physiological tests for elite athletes. London: Human Kinetics; 1991. p. 223-308.

13. Garrow JS, Webster J. Quetelet's index (WIH2) as a measure of fatness. Int J Obes. 1985;9(2):147-53.

14. Cole TJ, Bellizzi MC, Flegal KM, Dietz WH. Establishing a standard definition for child overweight and obesity worldwide: international survey. BMJ 2000;320:1240-3.

15. Pan H, Cole TJ. LMS Chartmaker. 2006. http://www. healthforallchildren.co.uk, accessed 11 march 2012.

16. Tudor-Locke C, Ainsworth BE, Adair LS, Popkin BM. Physical activity in Filipino youth: the Cebu Longitudinal Health and Nutrition Survey. Int J Obes Relat Metab Disord 2003; 27: 181_90.

17. Biddle SJH, Gorely T,Stensel DJ. Health-enhancing physical activity and sedentary behaviour in children and adolescents. J Sports Sci. 2004;22: 679-701.

18. Trost SG, Rosencrantz RR, Dzewaltowski D. Physical activity levels among children attending after school programs. Med Sci Sports Exercise 2008;40: 622-9.

19. Yungblut HE, Schinke RJ, McGannon KR. Views of adolescent female youth on physical activity during early adolescence. Sports Sci and Med. 2012;11: 39-50.

20. Pate RR, Dowda M, O'Neill JR, Ward DS. Change in physical activity participation among adolescent girls from 8th to 12th grade. J Phys Act Health 2007;4: 3-16.

21. Brooks, F. and Magnusson, J. Taking part counts: Adolscents' experiences of the transition from inactivity to active 
participation in school-based physical education. Health Education Res. 2006;21: 872-83.

22. Ferreira I, Van Der Horst K., Wendel-Vos W, Kremers S, Van Lenthe FJ, Brug, J. Environmental correlates of physical activity in youth: A review and update. Obesity Rev. 2007; 8:129-54.

23. Moore, J.B., Jilcott, S.B., Shores, K.A., Evenson, K.R., Brownson, R.C. and Novick, L.F. A qualitative examination of perceived barriers and facilitators of physical activity for urban and rural youth. Health Educ Res. 2010; 25: 355-67.

24. Santos MS, Hino AA, Reis RS, Anez CR. Prevalence of barriers for physical activity in adolescents. Rev Bras Epidemiol. 2010; 13:94-104.

25. Telama $R$, Yang X, Viikari J, Valimaki I,Wanne O, Raitakari O. Physical activity from childhood to adulthood: a 21year tracking study. Am J Prev Med. 2005; 28: 267-73.

26. Van de Laar RJ, Ferreira I, Mechelen WV, Prins MH, Twisk JW. Stehouwer CD. Lifetime vigorous but not light-tomoderate habitual physical activity impacts favorably on carotid stiffness in young adults: the amsterdam growth and health longitudinal study. Hypertension 2010; 55: 33-9.

27. Hallal PC, Gomez LF, Parra DC, Lobelo F, Mosquera J, Florindo $A A$, et al. Lessons learned after 10 years of IPAQ use in Brazil and Colombia. J Phys Act Health 2010; 7 (Suppl 2): 259-64.
28. Raustorp A, Ekroth Y. Tracking of pedometer-determined physical activity: a 10-year follow-up study from adolescence to adulthood in Sweden. J Phys Act Health. 2013; 10(8):1186-92

29. Martínez-Gómez D, Martínez-de-Haro V, Pozo T, Welk GJ, Villagra A, Calle $M E$, et al. Fiabilidad y validez del cuestionario de actividad física $P A Q-A$ en adolescentes españoles. Rev Esp Salud Pública. 2009; 83: 427-39.

30. Lima-Seerrano M, Lima-Rodriguez, Saez-Bueno A. Diseño y validación de dos escalas para medir la actitud hacia la alimentación y la actividad física durante la adolescencia. Rev Esp Salud Publica. 2012; 86(3): 253-68.

31. Urzúa A. Auto-reporte de salud y enfermedad en la adolescencia: Cuestionario CHIP-AE en adolescentes escolarizados chilenos. Rev Chil Pediatr. 2012; 83 (4): 336-44.

32. Goto R, Mascie-Taylor NCG. Precision of measurement as a component of human variation. J Physiol Anthropol. 2007; 26: 253-6.

33. Escalante Y. Actividad física, ejercicio físico y condición física en el ámbito de la salud pública. Rev Esp Salud Pública. 2011; 84(4): 325-8.

34. Moreno L, Concha F, Kain J. Intensidad de movimiento de escolares durante clases de educación física de colegios municipales: resultados según el profesional que efectúa las clases. Rev Chil Nutr. 2012;39(4):123-28. 